\title{
DELTA-NOTCH LATERAL INHIBITORY PATTERNING IN THE EMERGENCE OF CILIATED CELLS IN XENOPUS: EXPERIMENTAL OBSERVATIONS AND A GENE NETWORK MODEL
}

\author{
G. MARNELLOS \\ Sloan Center for Theoretical Neurobiology, \\ The Salk Institute for Biological Studies, San Diego, CA 92037, USA \\ and Institute for Neural Computation, UCSD, La Jolla, CA 92093, USA \\ G.A. DEBLANDRE \\ Molecular Neurobiology Laboratory, The Salk Institute for Biological Studies, \\ San Diego, CA 92037, USA \\ E. MJOLSNESS \\ Machine Learning Systems Group, Jet Propulsion Laboratory, \\ Pasadena, CA 91109, USA \\ C. KINTNER \\ Molecular Neurobiology Laboratory, The Salk Institute for Biological Studies, \\ San Diego, CA 92037, USA

\begin{abstract}
In diverse vert ebrate and invertebratesystems, lateral inhibition through the DeltaNotch signaling pathway can lead to cells in initially uniform epithelial tissues differentiating in "salt-and-pepper", regular spacing patterns. In this paper we examine lateral inhibition during the emergence of ciliated cells in Xenopus embryonic skin, using experimental manipulations of the Delta-Notch pathway and a connectionist gene-network model of the process. The results of our model are in agreement with previous models of regular patterning through lateral inhibition and reproduce the observations of our experimental assays. Moreover, the model provides an account for the variability of embryonic responses to the experimental assays, points to a component of lateral inhibition that may be the chief source of this variability, and suggests ways to control it. Our model could thus serve as a tool to generate predictions about this and other regular patterning systems governed by lateral inhibition.
\end{abstract}

\section{Introduction}

Lateral signaling through the Delta-Notch pathway has been implicated in many cell fate decisions across metazoans, during many stages of development and in many tissues ${ }^{1,2}$. Delta is a transmembrane protein that binds and activates its receptor, the transmembrane protein Notch, in neighboring cells. One type of activity ascribed to this pathway is lateral inhibition ${ }^{3,4,5,6,7}$ : cells that segregate from within a group of equipotential cells to adopt a particular fate use the pathway to prevent their neighbors from adopting the same fate; those neighbors are then diverted to a different fate or remain undetermined and competent to 
respond to subsequent inductive signals. Lateral inhibition through the DeltaNotch pathway is thought to be the mechanism that produces the more or less regular ("salt-and-pepper") spacing of differentiated cells in diverse systems, like for instance in vertebrate primary neurogenesis ${ }^{8,9}$ and hair-cell emergence in the inner ear ${ }^{10}$, or in the formation of sensory bristles, microchaetes, in Drosophila ${ }^{11}$.

Although Delta-Notch lateral inhibition has been extensively studied, its dynamics are not very well understood. In order to shed more light on this process and deduce its principle features, we have looked at lateral inhibitory patterning in a relatively simple and well accessible system: the differentiation of ciliated cells in a salt-and-pepper pattern in Xenopus embryonic epidermis (see Fig. 2A). These cells differentiate from within the uniform inner layer of the skin as ciliated cell precursors, a process that depends on Notch signaling, and then intercalate to the outer layer of the epidermis to form mature ciliated cells ${ }^{12}$.

We have examined the initial stage of this differentiation process (specification of cell fate within the inner epidermal layer) through experimental perturbations of the Delta-Notch pathway (injections of Delta or of a constitutively active form of Notch) and through a gene-network model of the process that is based on the connectionist framework of Mjolsness et al. ${ }^{13}$. We have previously used a similar modeling approach to simulate Delta-Notch lateral inhibition during neuroblast and sensory organ precursor differentiation in Drosophila ${ }^{14,15}$, where the Notch pathway was first characterized.

We have tuned the parameters of our model through optimization of an objective function that captures the pattern of emergence of ciliated cells in the biological system. Our model can reproduce the phenotypes observed experimentally under the assays we have applied. Statistical analysis of "genotypes" in the model suggests that the model can account for the variability of embryonic responses to the experimental assays and has highlighted a component of lateral inhibition that may be the chief source of this variability (thus yielding predictions as to how this variability may be controlled). Finally, our model results agree with previous models, analytical and computational, that have studied the formation of regular spacing patterns through lateral inhibition ${ }^{16,17}$.

\section{Model}

\subsection{Dynamics}

In our model the epidermal epithelium is represented as a hexagonal array of cells that express "genes" for Notch and Delta (see Fig. 1). Genes in the model interact as nodes in a recurrent neural net. A gene $a$ sums inputs from genes in the same cell and in neighboring cells at time $t$ according to

$$
u_{a}(t)=\sum_{b} T_{a b} v_{b}(t)+\sum_{i} \sum_{b} \hat{T}_{a b} \hat{v}_{b}^{i}(t)
$$


where $T$ is the matrix of cell-autonomous gene interaction strengths and the $v_{b}(t)$ are gene product levels within the cell; $\hat{T}$ is the matrix of lateral gene interactions with neighboring cells and the $\hat{v}_{b}^{i}(t)$ gene product levels in neighboring cell $i$. Each cell only interacts with its six immediate neighbors in the hexagonal lattice.

Levels $v_{a}(t)$ of gene products then change according to

$$
\frac{d v_{a}}{d t}=R_{a} g\left(u_{a}(t)+h_{a}\right)-\lambda_{a} v_{a}(t)
$$

where $u_{a}(t)$ is the linear sum of Eq. $1, g$ a sigmoid function given by $g(x)=$ $0.5\left(1+\frac{x}{\sqrt{\left(1+x^{2}\right)}}\right), R_{a}$ the rate of production of gene $a$ 's product, $h_{a}$ the threshold of activation of gene $a$ and $\lambda_{a}$ the rate of decay of gene $a$ product. For more details on this connectionist framework and its application to lateral inhibition models, see Mjolsness et al. ${ }^{13}$ and Marnellos $(1997)^{18}$.

Levels of gene products should be thought to correspond to gene product activities in the biological system rather than to actual concentrations, and gene interactions should be thought to correspond closer to genetic rather than specific biochemical (transcriptional etc.) interactions. And, of course, an interaction of two genes in the same cell will in general be different from an interaction of the same genes across cells; thus, for example, Delta may inhibit Notch activity in the same cell but increase Notch activity in neighboring cells. Initial conditions in the model are homogeneous: all cells start with the same levels of Notch and Delta with some gaussian noise, mean values of these levels are 10.0 for both Notch and Delta (see Fig. 1A); depending on the parameters of the model these levels may change in various ways over time. The numerical values of these levels are arbitrary, and only their relative changes over time matter.

The dynamics equations, Eq. 2, are integrated using Euler's method over the development time $\Omega$ ( $\Omega$ is 50 time steps in the work described here), and the phenotypes of the cells are assessed at the end of time $\Omega$. If at the end of the integration time a cell has high Delta levels (above a threshold of 10.0) and low Notch levels (below a threshold of 1.0), then it is considered to have differentiated as a ciliated cell, as is thought to be the case in the biological system (and in other Delta-Notch lateral inhibition systems). Similarly, if at the end a cell has high Notch levels (above 10.0) and low Delta levels (below 1.0), then it is considered to have differentiated as an epidermal cell. The specific numerical values of these thresholds are again arbitrary and are simply chosen to be of the same order of magnitude as the initial gene product levels.

\subsection{Objective function}

The parameters of the model are estimated using an objective function optimization approach, minimization in this case (see Eq. 3). One of the goals of the optimization is to have Notch and Delta levels in cells evolve in such a way over development time $\Omega$ that, at the end, a certain desired fraction ( 1 out of 3 , which 


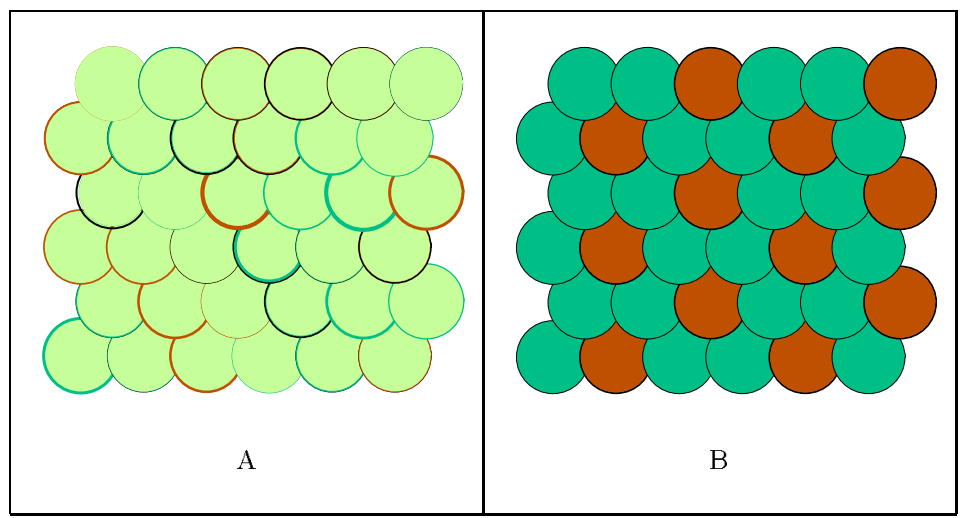

Figure 1: (A) Initial conditions in the model epithelium are homogeneous: all cells start with the same levels of Notch and Delta, with some gaussian noise. (B) Desired final pattern where a fraction of the cells ( 1 out of 3 ) have differentiated as ciliated cells, i.e. have high Delta expression (dark grey disks) and very low Notch, and the rest as epidermal cells with high Notch (lighter grey disks) and very low Delta. We have used periodic boundary conditions for our model epithelium.

is thought to be the fraction of ciliated cells in Xenopus) of the initially equivalent cells have differentiated as ciliated cells and the rest as epidermal cells (see Fig. 1B); this is captured in the DesiredFraction term of the objective function. The ciliated cells shouldn't have other ciliated cells as immediate neighbors, but should be surrounded by epidermal cells; this is expressed in the Repulsion term of the objective function. The objective function also has a (quadratic) penalty term, which penalizes parameter values so as to keep them bounded; parameters that are constrained to be positive or negative have a higher weighting in this term if they are not of the desired sign.

$$
\text { Objective Function }=w_{D} \cdot \text { DesiredFraction }+w_{R} \cdot \text { Repulsion }+w_{P} \cdot \text { Penalty }
$$

The terms in the objective function are weighted appropriately, so as to ensure that they all contribute significantly to the objective function; in the runs described here we have used $w_{D}=1.0, w_{R}=5.0, w_{P}=1.0$, but the precise values of these weights are not crucial.

We calculate the DesiredFraction term of the objective function as follows: for each cell $i$ in the epithelium we compute how close that cell is to being a ciliated cell or an epidermal cell, i.e. we compute its ciliarit $y_{i}$ and its epidermalit $y_{i}$, both of which depend on the levels of Notch and Delta in the cell and have the form

$$
\text { ciliarity } y_{i}=\prod_{q \in \text { genes }} \exp ^{-D_{i, q}^{4}}
$$

where $D_{i, q}$ is the difference of the level of gene $q$ in cell $i$ from the threshold 
for that gene in ciliated cells, as specified above; $D_{i, q}$ is set to zero if the gene level is on the desired side of the threshold and the value of the exponential in that case is 1 . epidermality $y_{i}$ is an analogous product as Eq. 4. Because of the almost step-like nature of these exponentials (which are close or at 1 if gene levels are close to or on the appropriate side of thresholds, and close to 0 if gene levels are away from thresholds on the wrong side) these products are essentially products of 1 and 0 and are 1 if a cell satisfies all the gene level requirements for being a ciliated cell (or, analogously, an epidermal cell). So ciliarity and epidermality can be used in an almost discrete way to count how many ciliated and epidermal cells have differentiated at the end, but they are still smooth differentiable functions which is useful for the optimization. Ciliarities and epidermalities of all $N$ cells in the epithelium are added up to give totalCiliarity $=\sum_{i}^{N}$ ciliarit $y_{i}$, totalEpidermality $=\sum_{i}^{N}$ epidermality $y_{i}$. The DesiredFraction term of the objective function is then a sum of the squared differences between the actual ciliated and epidermal cell numbers and the desired ones, diffC $=$ totalCiliarity - desiredCiliatedRatio $\cdot N$ and diff $E=$ totalEpidermality $-(1-$ desiredCiliatedRatio $) \cdot N$

$$
\text { DesiredFraction }=\operatorname{diff} C^{2}+\operatorname{diff} E^{2},
$$

and is minimized to 0 when the desired fraction of epithelium cells (one third) have differentiated as ciliated cells and all the rest as epidermal cells.

Finally the Repulsion term of objective function Eq. 3 is in essence the number of pairs of ciliated cells that are next to each other; this term is minimized when every ciliated cell is surrounded by non-ciliated cells

$$
\text { Repulsion }=\sum_{i \in \text { Cells }} \sum_{j \in \text { Surround }} \text { ciliarity }_{i} \cdot \text { ciliarity }_{j}
$$

where Cells are all cells of the epithelium and Surround the set of six immediate neighbors of cell $i$.

For the minimization of the objective function we have used simulated annealing. There are eight (8) parameters optimized on (the free parameters): production and decay rates of gene products, i.e. the $R_{a}$ 's and $\lambda_{a}$ 's of Eq. 2 (thresholds $h_{a}$ are not optimized on but have the value -1.5 , which ensures a low level of constitutive activation under the sigmoid activation function used); and elements of the cell-autonomous and lateral interaction matrices, $T$ and $\hat{T}$, indicated in the matrices below with a diamond. We have only included regulatory gene interactions for which there is some biological evidence $e^{7,1,2}$ and set the rest to zero. For a description of the simulated annealing algorithm used see Chapter 2 in Marnellos (1997) ${ }^{18}$ and references therein. Optimization is computationally expensive, so optimization runs were carried out on a $6 x 6$ cell array, as appears in Fig. 1. Tests of the resulting solutions, on the other hand, were carried out on larger arrays, as in Fig. 3. In both cases we imposed periodic 
boundary conditions.

\begin{tabular}{|c|c|c|}
\hline \multicolumn{3}{|c|}{ Cell-autonomous Interactions } \\
\hline \hline & Delta & Notch \\
\hline Delta & $\bullet$ & $\bullet$ \\
\hline Notch & $\bullet$ & \\
\hline
\end{tabular}

\begin{tabular}{|c|c|c|}
\hline \multicolumn{3}{|c|}{ Lateral Interactions } \\
\hline \hline & Delta & Notch \\
\hline Delta & & \\
\hline Notch & $\bullet$ & \\
\hline
\end{tabular}

\section{Experimental Observations}

In order to examine the role of the Delta-Notch pathway in the emergence of ciliated cell precursors, we looked at the expression of X-Delta-1 (a putative ligand of Notch in Xenopus and homolog of Drosophila Delta) by in situ hybridization, and also used RNA transcripts known to activate or inhibit Notch signaling. Specifically we have injected RNA transcripts encoding either NotchICD (which is the intracellular domain of Notch and constitutively activates Notch target genes) or X-Delta-1 into one blastomere of two-cell stage embryos, together with tracer $n$ LacZ RNA. These injections thus affect only one side of embryos, the other serving as control. We assessed the effects of the injections by in situ hybridization for $\alpha$-tubulin, an early marker of ciliated cell precursors. Embryo collections, RNA injections and in situ hybridizations were performed as described in Chitnis et al. ${ }^{8}$ and Deblandre et al. ${ }^{12}$

We examined X-Delta-1 expression in non-neural ectoderm, where ciliated cells are expected to emerge. X-Delta- 1 is expressed in scattered cells in the ventral ectoderm, a pattern that prefigures the expression of $\alpha$-tubulin, an early marker of ciliated cells; this suggests that cells expressing high levels of X-Delta1 differentiate as ciliated cells (which has been an assumption of our model). Injection of embryos with Notch-ICD RNA resulted in the complete loss of ciliated cell precursors, while injection of X-Delta-1 RNA increased the density of the precursors in only a fraction of embryos and the rest were unaffected, i.e. the penetrance of this assay was low. Our injection assay results are summarized in Fig. 2.

\section{Simulation Results}

We have run optimizations as described in the Model section above; the production and decay rates of gene products were constrained to be positive (and in the case of the decay rates between 0 and 1 ); in some of the runs the two crossdiagonal cell-autonomous interactions were constrained to be negative and the lateral interaction positive, as biological evidence suggests ${ }^{7,1,2}$. Under these conditions, optimization (simulated annealing) runs have very robustly minimized the objective function to almost zero (order of $10^{-3}$, not exactly zero of course because of the penalty term on the parameter values, which never becomes zero) 


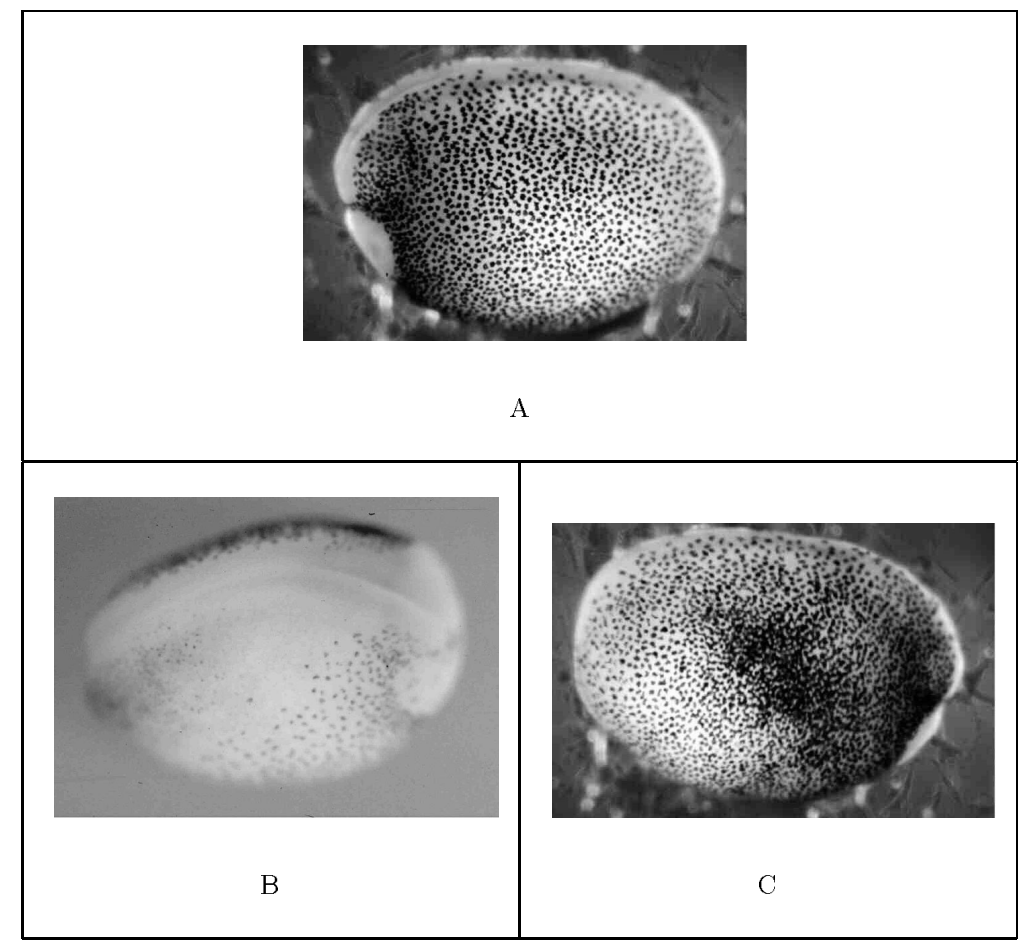

Figure 2: Stage 17-18 Xenopus embryos labeled by in situ hybridization for $\alpha$-tubulin, an early marker of ciliated cell precursors. (A) Untreated side of an embryo shows the regular spacing pattern of ciliated cell precursors in the epidermis (this is the control side of the embryo in panel C). (B) Embryo injected with constitutively active Notch (Notch-ICD) RNA and (C) embryo injected with X-Delta-1 RNA. Compare with non-injected embryos in panel A: Notch-ICD suppresses the emergence of ciliated cells, while overexpression of X-Delta-1 leads to greatly increased density of ciliated cells.

and returned sets of (free) parameter values, referred to here as solutions or genotypes, that are very similar to each other, i.e. have the same signs and orders of magnitude, and produce the same regular differentiation pattern; in these solutions, the unstable homogeneous initial state leads to a stable inhomogeneous differentiated state (see Fig. 1); for the desired ciliated cell ratio in these runs (1 out of 3 ) and for the size of hexagonal cell array $(6 \times 6)$ used in the optimization runs, this differentiation pattern is a global minimum of the objective function. The values of one of these solutions (genotypes) are presented, as an example, below.

\begin{tabular}{|c|c|c|c|}
\hline$R_{\text {Delta }}$ & $\lambda_{\text {Delta }}$ & $R_{\text {Noteh }}$ & $\lambda_{\text {Noteh }}$ \\
\hline 8.09 & 0.822 & 6.40 & 0.484 \\
\hline
\end{tabular}




\begin{tabular}{|c|c|c|}
\hline \multicolumn{3}{|c|}{ Cell-autonomous Interactions } \\
\hline \hline & Delta & Notch \\
\hline Delta & 1.38 & -1.70 \\
\hline Notch & -4.51 & \\
\hline
\end{tabular}

\begin{tabular}{|l|c|c|}
\hline \multicolumn{3}{|c|}{ Lateral Interactions } \\
\hline \hline & Delta & Notch \\
\hline Delta & & \\
\hline Notch & 9.93 & \\
\hline
\end{tabular}

To further analyze the properties of the genotypes that result from the optimization runs, we randomly generated genotypes as follows: we used simulated annealing at low temperature to generate genotypes by tweaking the 8 free parameters one at a time; we then selected those genotypes that (a) gave objective function values very close to the global minimum (which, as was mentioned above, was globalMin $\approx 10^{-3}$ ), within a margin of $M=10.0$ (half of this margin, i.e. 5.0, was for the penalty term), and (b) differed from the previous selected genotype in the sequence by at least $10 \%$ in one of the free parameter values.

We thus came up with 486 genotypes that all essentially gave the regular spacing differentiation pattern (Fig. 1B and Fig. 3A) under unperturbed conditions, and proceeded to test them with the Notch and Delta injection assays. The constitutively active Notch (Notch-ICD) assay in the model consisted of and extra constant negative input to the Delta gene of magnitude $i c d=-10.0$ in all cells of the epithelium (i.e. an extra input icd to the sum of Eq. 1 for Delta). The Delta injection in a patch of epithelium cells was simulated by increasing the initial Delta levels of cells in the patch 10-, 100- or 1000-fold.

We didn't individually check the 486 genotypes with the Delta and NotchICD injection assays. Instead we grouped them into 10 clusters of similar genotypes by K-means clustering (SPSS 8.0 package) and examined the cluster center responses to the assays. All cluster centers responded the same way to the NotchICD injection: in all cases ciliated cell differentiation was abolished (Fig. 3C), which is also what we observed experimentally. By contrast, Delta injections produced a patch of ciliated cells at the place of the injection for some of the cluster centers (Fig. 3B), while in others injections did not affect the emergence of ciliated cells in a regular spaced pattern (Fig. 3A). This observation concurs with the experimental finding that Delta injections produce the ciliated cell patch phenotype in only part of the embryos and the rest are not affected, as reported above. Clusters with this response to the Delta injection (Patch phenotype) were lumped together and clusters which developed the regular spaced pattern (NoPatch phenotype) were also lumped together, resulting in two bigger clusters depicted in Fig. 3D.

Principal components analysis of the genotypes reveals 4 components (see Table 1) that account for $75 \%$ of the variance (the first three of those account for $65 \%$ of the variance). The decay and production rates of Notch contribute most heavily, with opposite signs, to the first principal component, which can be therefore thought to express the amount of available Notch; this component 


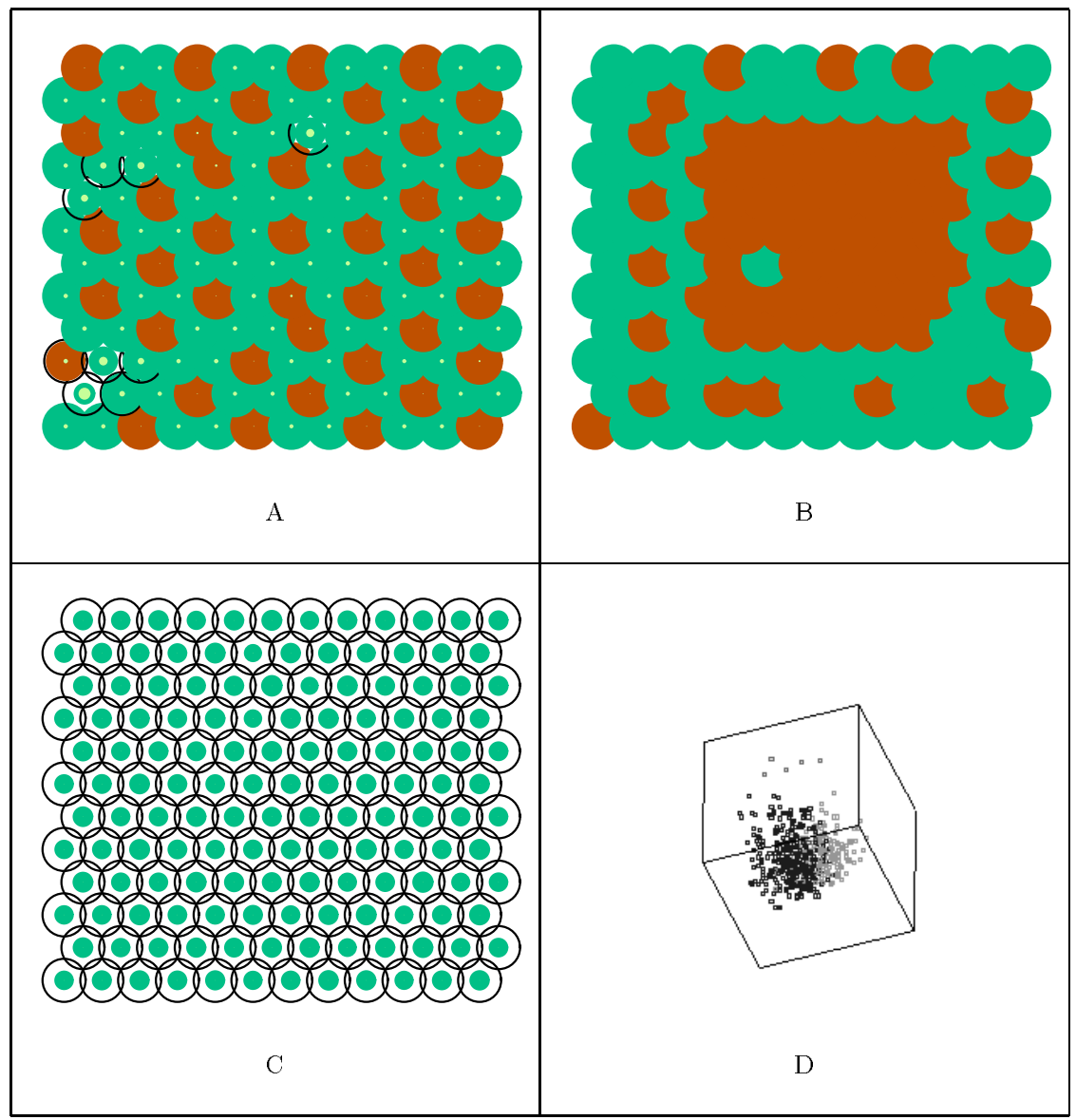

Figure 3: (A) The regular spacing pattern of differentiation of ciliated cells in untreated cell arrays. The solution (shown in Results) was obtained by optimization on a $6 \times 6$ array of cells, as described in Methods, and applied to a $12 \times 12$ array. The same pattern appears in the cluster of genotypes that are not affected by the Delta injection. Delta expression is depicted by dark grey disks and Notch expression by light grey disks (as in Fig. 1). (B) Delta injection, simulated by a manyfold increase of initial Delta levels in a patch of cells, leads to an increased density of ciliated cells in the patch; the effect is observed in a fraction of the genotypes, the rest are not affected by the Delta injection (see panel A), which agrees with experimental observations. (C) Expression of constitutively active Notch (Notch-ICD), simulated in the model by a constant negative input to the Delta gene, totally supresses the emergence of ciliated cells, as observed experimentally. (D) Genotypes are depicted as darker grey or lighter grey depending on their phenotype for Delta injections; lighter grey denotes genotypes which developed the regularly spaced differentiation pattern of ciliated cells despite the injection (NoPatch cluster), and darker grey the genotypes that developed a patch of ciliated cells at the place of the injection (Patch cluster). The axes are the first three components (rotated) of the principal components analysis of the genotypes. 
accounts for most of the variance, nearly one third, which is consistent with the biology of the system, as Notch is expressed ubiquitously and the differentiation of ciliated cells seems to be driven instead by Delta.

Variance along the third principal component (the left-right axis in Fig. 3D) seems to correlate with the variance in the Delta injection phenotype (Patch or NoPatch); this component balances cell-autonomous inhibition of Notch by Delta, $T_{\text {Notch-Delta }}$, with the activation of Notch by Delta across cells, $\hat{T}_{\text {Notch-Delta }}$ (and, to a lesser extent, with the levels of available Delta). NoPatch genotypes have on average higher absolute values for $T_{\text {Noteh-Delta }}$ and $\hat{T}_{\text {Notch-Delta }}$ and a lower Delta production rate than Patch genotypes; the model would therefore predict that embryo perturbations that increased cell-autonomous inhibition and lateral activation of Notch by Delta and decreased the production rate of Delta would tend to make embryos adopt the NoPatch phenotype (i.e. become insensitive to Delta injections).

\begin{tabular}{|l|r|r|r|r|}
\hline & \multicolumn{4}{|c|}{ Components } \\
\cline { 2 - 5 } & 1 & 2 & 3 & 4 \\
\hline$\lambda_{\text {Notch }}$ & $-\mathbf{0 . 8 9 5}$ & & & \\
$R_{\text {Notch }}$ & $\mathbf{0 . 8 8 8}$ & -0.207 & & \\
$T_{\text {Delta-Notch }}$ & & $\mathbf{0 . 8 9 0}$ & & \\
$T_{\text {Delta-Delta }}$ & 0.272 & $\mathbf{- 0 . 7 2 2}$ & & \\
$T_{\text {Notch-Delta }}$ & 0.243 & & $\mathbf{- 0 . 8 5 8}$ & \\
$\hat{T}_{\text {Notch-Delta }}$ & & 0.301 & $\mathbf{0 . 6 9 1}$ & \\
$R_{\text {Delta }}$ & & 0.257 & 0.302 & $\mathbf{0 . 8 1 2}$ \\
$\lambda_{\text {Delta }}$ & & & 0.272 & $\mathbf{- 0 . 6 8 7}$ \\
\hline
\end{tabular}

Table 1: Rotated principal components matrix with coefficients of the genotype parameters on each of the components. The 8 parameters are the free parameters listed in Methods. Extraction of the components and rotation (quartimax with Kaiser normalization) were performed with the statistical package SPSS 8.0. The parameters' highest coefficients on each component are shown in bold. Only coefficients of absolute value 0.2 or larger are shown.

\section{Discussion}

In the work presented in this paper we have examined the differentiation of ciliated cells in Xenopus embryos by perturbing the Delta-Notch signaling pathway, and by constructing a gene-network model of the process. In our experiments, we have injected embryos with a constitutively active form of Notch (NotchICD) and with X-Delta-1. Our model has reproduced the phenotypes of both untreated and injected embryos. Notch-ICD injections abolished the differentiation of ciliated cells, and this was reflected in the model. Delta injections increased the density of ciliated cells in only some of the embryos and didn't seem to affect others, which was also the case in the model. Although we cannot 
yet rule out other factors that may cause this variability (for instance, factors that have to do with the exact location of the RNA injection, something hard to control), our model does suggest that the observed low penetrance of the Delta assay may be due to natural genetic variation in the embryos and/or differences in their lateral inhibition physiology. And principal components analysis of genotypes generated in the model has pointed to a specific component of lateral inhibition as the source of the observed variability; this prediction could help test the validity of the model.

The model presented here has used a similar connectionist approach as our models of lateral inhibition in Drosophila neurogenesis ${ }^{14,15}$. In order to make the model more interpretable, we have tried to limit the number of free parameters as much as possible, e.g. by setting some regulatory gene interactions, for which there is not much biological evidence, to zero. It's not clear though if the number of parameters used in this model is the minimum one necessary for the phenomena described here. We have also constrained the signs of gene interactions if there was evidence for that; this has not necessarily made the optimization task easier: it may have restricted the search space, but may have also imposed obstacles to moving around in that space. The objective function used here to estimate the parameters of the model is new (in previous work we had instead used fits to gene expression data ${ }^{14,15}$ ); as the properties of its global optimum are known, this objective function has been a good test of the performance of our stochastic optimization algorithm on gene networks; it has demonstrated that simulated annealing can consistently converge to the global optimum in this problem.

Our model is similar in structure to Collier et $a l^{16}$ with the difference that we have included more cell-autonomous interactions than they have. These extra interactions may have allowed our model to capture the injected embryo phenotypes; the Collier et al. model dealt with unperturbed patterns, and it would be interesting to see how it would behave under the perturbations described in this paper. The two models differ in their mathematical detail, but the patterns that emerge both in our model and theirs seem to be insensitive to the precise values of model parameters. In their model as in ours, the homogeneous initial state is unstable and through similar dynamics leads to a stable heterogeneous differentiated state.

The 1:3 (ciliated:epidermal) spacing of ciliated cells in Xenopus is a spacing that has frequently appeared in models of lateral inhibition ${ }^{17,16}$, both in regular hexagonal and non-regular polygonal cell lattices ${ }^{17}$, so perhaps this is one of the reasons that optimizations in our model have so robustly converged to that desired solution. We should try other spacings in our model to see how readily the lateral inhibition system can produce them and how the genotypes for those differ from the genotypes that produce the 1:3 spacing. We might then look experimentally in embryos for perturbations that can alter ciliated cell precursor spacing. 
In conclusion, the model presented here appears to be a good tool for the study of Xenopus ciliated cell differentiation or of other similar systems where differentiation in regular spacing patterns is governed by lateral inhibitory signaling. One such system is primary neurogenesis in vertebrates ${ }^{8}$, which has many similarities with the ciliated cell system but also differences - for instance Delta injections have a different effect there, namely they suppress the differentiation of neurons instead of promoting it. It would be interesting to use the model to tease out the components of lateral signaling that underlie similarities and differences between the two systems.

\section{Acknowledgements}

G.M. and E.M. were supported in part by Office of Naval Research grant N0001497-1-0422. C.K. and G.A.D were supported by a grant of the N.I.H. and by a fellowship of the Belgian-American Educational Foundation to G.A.D.

\section{References}

1. J. Lewis, Seminars in Cell and Developmental Biology 9, 583 (1998).

2. S. Artavanis-Tsakonas et al., Science 284, 770 (1999).

3. B. Wigglesworth, V, Journal of Experimental Zoology 17, 180 (1940).

4. C. Stern, American Scientist 42, 213 (1954).

5. P. Simpson, Development 109, 509 (1990).

6. M. Muskavitch, Developmental Biology 166, 415 (1994).

7. S. Bray, Seminars in Cell and Developmental Biology 9, 591 (1998).

8. A. Chitnis et al., Nature 375, 761 (1995).

9. C. Haddon et al., Development 125, 359 (1998).

10. J. Adam et al., Development 125, 4645 (1998).

11. P. Heitzler and P. Simpson, Cell 64, 1083 (1991).

12. G. Deblandre et al., A two-step mechanism generates the spacing pattern of ciliated cells in the skin of Xenopus embryos, submitted.

13. E. Mjolsness et al., Journal of Theoretical Biology 152, 429 (1991).

14. G. Marnellos and E. Mjolsness, in Pacific Symposium on Biocomputing, volume 3, 30-41 (1998).

15. G. Marnellos and E. Mjolsness, in Artificial Life VI, Proceedings of the Sixth International Conference on Artificial Life, 161-170 (1998).

16. J. Collier et al., Journal of Theoretical Biology 183, 429 (1996).

17. M. Tanemura et al., Journal of Theoretical Biology 153, 287 (1991).

18. G. Marnellos, Gene Network Models Applied to Questions in Development and Evolution, Ph.D. thesis, Yale University (1997). 